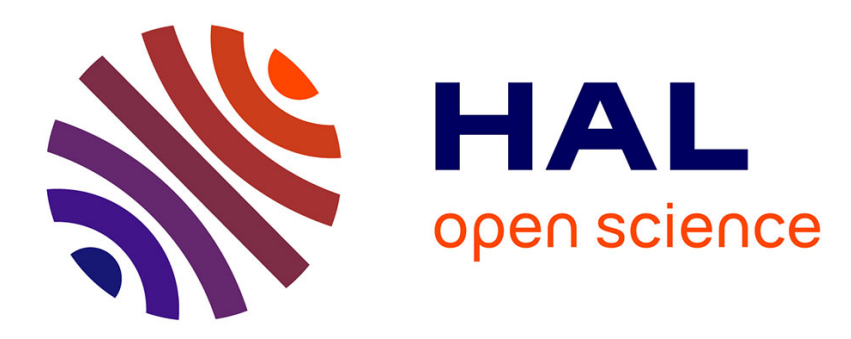

\title{
On the Angular Resolution Limit Uncertainty Under Compound Gaussian Noise
}

\author{
Maria S Greco, Remy Boyer
}

\section{To cite this version:}

Maria S Greco, Remy Boyer. On the Angular Resolution Limit Uncertainty Under Compound Gaussian Noise. Signal Processing, 2019, 164, pp.217-224. hal-02144024

\section{HAL Id: hal-02144024 \\ https://hal.science/hal-02144024}

Submitted on 29 May 2019

HAL is a multi-disciplinary open access archive for the deposit and dissemination of scientific research documents, whether they are published or not. The documents may come from teaching and research institutions in France or abroad, or from public or private research centers.
L'archive ouverte pluridisciplinaire HAL, est destinée au dépôt et à la diffusion de documents scientifiques de niveau recherche, publiés ou non, émanant des établissements d'enseignement et de recherche français ou étrangers, des laboratoires publics ou privés. 


\title{
On the Angular Resolution Limit Uncertainty Under Compound Gaussian Noise
}

\author{
Maria S. Greco and Remy Boyer
}

\begin{abstract}
The Angular Resolution Limit (ARL) is a fundamental statistical metric to quantify our ability to resolve two closely-spaced narrowband far-field complex sources. This statistical quantity, is defined as the minimal angular deviation between the two sources to be separated for a prefixed detection-based performance. In this work, we assume that the sources of interest are corrupted by a compound-Gaussian noise. In the standard literature, denoting with $\delta$ the true distance between the two sources, the derivation of the ARL is based on the statistical distribution of the Generalized Likelihood Ratio Test (GLRT) for a binary test where there is only one source under the null hypothesis (i.e., $\delta=0$ ) and two sources under the alternative hypothesis $\delta \neq 0$. In literature, the true angular distance (TAD) is generally considered as an unknown deterministic parameter, then a maximum likelihood-based estimation of $\delta$ is exploited in the GLRT. In this paper, breaking away from existing contributions, we suppose that the TAD is a random variable, Gaussian distributed, meaning that $\delta \sim \mathcal{N}\left(\delta_{0}, \sigma_{\delta}^{2}\right)$. The TAD uncertainty can have many causes as for instance moving sources or/and platform, antenna calibration error, etc. In this work, a generic and flexible (but common) statistical model of the uncertain knowledge of the TAD is preferred instead of a too much specified error model. The degree of randomness (or uncertainty) is quantified by the ratio $\xi=\delta_{0}^{2} / \sigma_{\delta}^{2}$. The standard framework of the GLRT is no longer feasible for our problem formulation. To cope with the compound Gaussian noise modeling and the random model of the TAD, a powerful upper bound from information/geometry theory is exploited in this work. More precisely, a new expected Chernoff Upper Bound (CUB) on the minimal error probability is introduced. Based on the analysis of this upper bound, we show that the expected-CUB is highly dependent on the degree of uncertainty, $\xi$. As a by-product, the optimal $s$-value in the Chernoff divergence for which the expected-CUB is the tightest upper bound is analytically studied and the role of the mean value $\delta_{0}$ in the ARL context is analyzed.
\end{abstract}

\section{INTRODUCTION}

Estimator-independent performance metrics are essential and powerful statistical tools to study a sensing system. Maybe the most popular bounds are due to the pioneer work of Cramér and Rao [27] in an estimation point of view and due to Smith in the detection/resolution perspective [1]. Such quantities allows to $(i)$ characterize the highest estimation/detection accuracy than we can hope to reach, ( $i i)$ avoid costly Monte-Carlo simulations dedicated to a particular estimator, (iii) identify the key model parameters involved in the design of the sensing system and $(i v)$ get more insight into the physic of the underlying problem. It is worth noting that the two contexts are in fact closely related as demonstrated in [4].

The resolvability of closely spaced signals, in terms of parameter of interest, for a given scenario (e.g., for a given Signal-to-Noise Ratio - SNR -, a given number of snapshots and/or a given number of sensors) is a former and challenging problem which was recently updated by Smith [1], Liu and Nehorai [4], Amar and Weiss [2], 
Sharman and Milanfar [13]. More precisely, the concept of statistical Angular Resolution Limit (ARL), i.e., the minimum distance between two closely spaced signals embedded in an additive noise that allows a correct resolvability/parameter estimation, is rising in several applications especially in problems such as array processing [9,10,12], MIMO radar [6]-[8], multidimensional harmonic estimation [11] or dictionary learning [23]. In the literature, several authors have focused their effort on the study of the Fisher information [24]-[26], but in most of the contributions, the ARL is derived from the asymptotic performance of the Generalized Likelihood Ratio Test (GLRT) where the unknown deterministic angular distance between the two sources has been replaced by its Maximum Likelihood (ML) estimate. Specifically, the GLRT is considered for a binary test where there is only one source under the null hypothesis (i.e., $\delta=0$ ) and two sources under the alternative hypothesis $\delta \neq 0$.

When the number of measurements grows, the MSE between the estimated angular distance and the TAD decreases to zero at the rate of the deterministic Cramér-Rao Bound (CRB) [22]. This partially explains why the "separation" of the distributions of the GLRT under the two hypotheses is completely characterized by the value of the noncentrality parameter which is a function of the TAD, the array manifold and the SNR. This framework is based on an ideal knowledge of the model and on an infinite number of measurements. We think that there is a lack of contributions on the more realistic problem where the knowledge of the TAD is uncertain. To the best of our knowledge, only [12] tackles this problem. Indeed, the ARL is derived in the context of an uncertain steering matrix. In other words, this model-based analysis is limited to a class of array model error and cannot take into account other causes of error as for instance on the measured sources. Finally, in [3] the sources are assumed to be stochastic. This means that the mathematical formalism of the hypothesis test could be close to the one used in this work. But, as a major difference with the proposed methodology, the TRL in [3] is a deterministic quantity. Breaking away from existing contributions, in this work, a more generic and flexible model of error is considered. Specifically, we suppose that the true angular distance (TAD) $\delta$ between the two sources is not deterministic, but random, Gaussian distributed, such that $\delta \sim \mathcal{N}\left(\delta_{0}, \sigma_{\delta}^{2}\right)$. The randomness of $\delta$ can be due to many factors, as, for instance, the non-perfectly compensated slow movement of the receiver platform, or of the sources or both. Consequently, the distance changes from one observation to the other. We adopted here the Gaussian model for the randomness of $\delta$, as usual for the observation error in radar tracking scenarios [15]. The degree of randomness or of uncertainty is quantified by the ratio $\xi=\delta_{0}^{2} / \sigma_{\delta}^{2}$. Indeed, for $\xi \rightarrow \infty, \delta \rightarrow \delta_{0}$ and it tends to be deterministic. On the contrary, for $\xi \rightarrow 0$, our degree of uncertainty tends to be maximal. The detection performance for a random quantity in terms of minimal error probability is often analytically intractable [14], particularly if the useful signal is embedded in non-Gaussian noise. To alleviate this technical difficulty, we exploit some powerful tools from the information theory $[17,18]$ and in particular the Chernoff Upper Bound (CUB) on the minimal error probability $[16,17]$. More precisely, the class of compound-Gaussian noise is of interest in this work due to its ability to accurately model physical phenomena in the context of several important applications as for instance [19]-[21]. To cope with this difficult context, a new expected CUB is introduced, derived and studied. To the best of our knowledge, only the single contribution [28] derives the ARL for non-Gaussian disturbance, i.e., in the MIMO radar context with K-distributed clutter. Our work has two main differences. First, our approach of the problem is developed in the information geometric framework. The second main difference resides on the fact that the TAD 
is considered random.

The remainder of this paper is organized as follows. In section 2, the array processing model and the linearized hypothesis test are introduced. In Section 3, after a brief presentation of the the state-of-art, the detection problem at hand for the considered framework is presented. In particular, the Chernoff Upper Bound (CUB) is derived in both Gaussian and compound-Gaussian cases. In section 4 some results are shown and commented, and final conclusions are given. The appendix contains the formalism of the CUB tacking into account random nuisance parameters.

\section{ARRAY PROCESSING MODEL AND LINEARIZED HYPOTHESIS TEST}

\section{A. Array processing model}

We consider the context of two probing sources far-field narrowband deterministic sources denoted by $s_{1}(k)$ and $s_{2}(k)$ where $k$ stands for the $k$-th snapshot with $s_{1}(k) \neq s_{2}(k)$. Probing sources are known at the emitter (see for instance [31,32]). The two source vectors $\mathbf{s}_{1}$ and $\mathbf{s}_{2}$ are collected over $K$ snapshots on a $L$ sensors array . Each source is associated to a steering vectors denoted by $\mathbf{a}\left(\omega_{1}\right)$ and $\mathbf{a}\left(\omega_{2}\right)$ characterized by two unknown angular frequencies, denoted by $\omega_{1}$ and $\omega_{2}$, respectively. Consequently, the observation vector for the $k$-th snapshots on the $L$ sensors of the array is given by

$$
\mathbf{y}(k)=\mathbf{a}\left(\omega_{1}\right) s_{1}(k)+\mathbf{a}\left(\omega_{2}\right) s_{2}(k)+\mathbf{w}(k)
$$

where $\mathbf{w}(k)$ is the additive noise for the $k$-th snapshot. For $K$ observation vectors, we obtain the following $(K L) \times 1$ long vector:

$$
\mathbf{y}=\left[\begin{array}{lll}
\mathbf{y}(1)^{T} & \ldots & \mathbf{y}(K)^{T}
\end{array}\right]^{T}=\mathbf{a}\left(\omega_{1}\right) \otimes \mathbf{s}_{1}+\mathbf{a}\left(\omega_{2}\right) \otimes \mathbf{s}_{2}+\mathbf{w}
$$

where $\otimes$ stands for the Kronecker product and $\mathbf{w}$ is the $(K L) \times 1$ noise vector. The statistical noise model will be discussed in the sequel.

\section{B. Formulation of the detection test}

Let $\delta=\omega_{2}-\omega_{1}$ be the TAD between the two sources. The problem of resolving two closely spaced sources can be formulated as a binary hypothesis test as follows:

$$
\left\{\begin{array}{l}
\mathcal{H}_{0}: \quad \delta=0, \quad \text { "only one source is detected at } \omega_{c} ", \\
\mathcal{H}_{1}: \quad \delta \neq 0, \quad \text { "two sources are detected with different angular-frequencies". }
\end{array}\right.
$$

The hypothesis $\mathcal{H}_{0}$ represents the case where the two sources exist but are combined into a single signal, whereas the alternative hypothesis $\mathcal{H}_{1}$ embodies the situation where the two sources can be separated.

The scenario where the two sources are largely separated in angle is meaningless since our aim is to evaluate the smallest AD. So, it makes sense to assume that the two sources are closely-spaced in angle. In other words, assuming a small $\delta$ is a realistic assumption meaning that terms $O\left(\delta^{2}\right)$ are discarded from our analysis. Let $\omega_{c}=\frac{\omega_{1}+\omega_{2}}{2}$ be the known central angular-frequency. This "physical" assumption allows to considerably simplify the mathematical 
formulation of the problem of interest. Indeed, recall that $\mathbf{a}\left(\omega_{1}\right)=\mathbf{a}\left(\omega_{c}-\frac{\delta}{2}\right)$ and $\mathbf{a}\left(\omega_{2}\right)=\mathbf{a}\left(\omega_{c}+\frac{\delta}{2}\right)$ are two non-linear functions of $\delta$ but after linearization around $\omega_{c}$, we obtain two nice relations linear in $\delta$ such as

$$
\begin{aligned}
& \mathbf{a}\left(\omega_{1}\right)=\stackrel{1}{\approx} \mathbf{a}\left(\omega_{c}\right)-\frac{j}{2} \delta \dot{\mathbf{a}}\left(\omega_{c}\right)+O\left(\delta^{2}\right), \\
& \mathbf{a}\left(\omega_{2}\right)=\stackrel{1}{\approx} \mathbf{a}\left(\omega_{c}\right)+\frac{j}{2} \delta \dot{\mathbf{a}}\left(\omega_{c}\right)+O\left(\delta^{2}\right),
\end{aligned}
$$

where symbol $\stackrel{1}{\approx}$ stands for first-order approximation and $\dot{\mathbf{a}}\left(\omega_{c}\right)=-\left.j \frac{\partial \mathbf{a}(\omega)}{\partial \omega}\right|_{\omega=\omega_{c}}$. It is straightforward to obtain the linear approximation of model (2) [10]:

$$
\mathbf{y} \stackrel{1}{\approx} \boldsymbol{\mu}_{\delta}+\mathbf{w}
$$

where $\boldsymbol{\mu}_{\delta}=\mathbf{a}\left(\omega_{c}\right) \otimes\left(\mathbf{s}_{1}+\mathbf{s}_{2}\right)+\frac{j}{2} \delta \dot{\mathbf{a}}\left(\omega_{c}\right) \otimes\left(\mathbf{s}_{2}-\mathbf{s}_{1}\right)$.

Discarding the known signals, we define the new observation vector $\mathbf{z}=\mathbf{y}-\mathbf{a}\left(\omega_{\mathbf{c}}\right) \otimes\left(\mathbf{s}_{\mathbf{1}}+\mathbf{s}_{\mathbf{2}}\right)$. In this case the hypothesis test (3) becomes

$$
\begin{cases}\mathcal{H}_{0}: & \mathbf{z}=\mathbf{w} \\ \mathcal{H}_{1}: & \mathbf{z}=\delta \mathbf{p}+\mathbf{w}\end{cases}
$$

where $\mathbf{p}=\frac{j}{2} \dot{\mathbf{a}}\left(\omega_{c}\right) \otimes\left(\mathbf{s}_{2}-\mathbf{s}_{1}\right)$

\section{NEW PROPOSED FRAMEWORK: UNCERTAIN TAD IN COMPOUND GAUSSIAN NOISE}

\section{A. Brief description of the state-of-art}

In the sequel, we briefly recall two standard methodologies in the case of a deterministic unknown TAD in white Gaussian noise and in colored signal-dependent noise. The purpose of these two paragraphs is to point out the limitations of the existing methods.

1) Deterministic unknown TAD in white Gaussian noise: In the standard literature (see for instance [10]), the binary hypothesis test is

$$
\mathbf{z} \sim\left\{\begin{array}{l}
p_{0}(\mathbf{z})=\mathcal{C N}\left(\mathbf{0}, \sigma^{2} \mathbf{I}\right) \\
p_{1}(\mathbf{z} ; \delta)=\mathcal{C N}\left(\delta \mathbf{p}, \sigma^{2} \mathbf{I}\right)
\end{array}\right.
$$

The GLRT is defined as

$$
T(\mathbf{z})=\log \frac{\max _{\delta \in \mathbb{R}} p_{1}(\mathbf{z} ; \delta)}{p_{0}(\mathbf{z})} .
$$

The MLE integrating the constraint of a real $\delta$ of the TAD is given by $[7,10]$ :

$$
\hat{\delta}(\mathbf{z})=\frac{\Re\left\{\mathbf{p}^{H} \mathbf{z}\right\}}{\|\mathbf{p}\|^{2}} .
$$

Using the above expression in $T(\mathbf{z})$, and if $K L \rightarrow \infty$,

$$
T(\mathbf{z}) \sim \begin{cases}\mathcal{H}_{0}: & \chi_{1} \\ \mathcal{H}_{1}: & \chi_{1}\left(\lambda\left(P_{f a}, P_{d}\right)\right)\end{cases}
$$


when $\chi_{1}$ (resp. $\left.\chi_{1}\left(\lambda\left(P_{f a}, P_{d}\right)\right)\right)$ denotes the centered (resp. non-central) Chi-squared distribution with a single degree of freedom [14] characterized by the probabilities of false alarm $P_{f a}$ and of detection $P_{d}$. The ARL is given by

$$
\delta_{A R L}=\frac{\sqrt{2} \sigma \sqrt{\lambda\left(P_{f a}, P_{d}\right)}}{\left\|\mathbf{s}_{2}-\mathbf{s}_{1}\right\|\left\|\dot{\mathbf{a}}\left(\omega_{c}\right)\right\|}
$$

After setting the desired performance of the test $\left(P_{f a}\right.$ and $\left.P_{d}\right)$, the above result shows that the ARL is derived as a function of the true source vectors, the ideal array manifold and for $K L \rightarrow \infty$.

2) Deterministic unknown TAD in signal-dependent noise: In [12], we assume that the steering vectors are corrupted by an additive error, $\mathbf{e}_{i} \sim \mathcal{C N}\left(\mathbf{0}, \sigma_{e}^{2} \mathbf{I}\right)$, such as

$$
\tilde{\mathbf{a}}\left(\omega_{i}\right)=\mathbf{a}\left(\omega_{i}\right)+\mathbf{e}_{i}, \quad 1 \leq i \leq 2
$$

So, the array processing model was

$$
\mathbf{y}=\mathbf{a}\left(\omega_{1}\right) \otimes \mathbf{s}_{1}+\mathbf{a}\left(\omega_{2}\right) \otimes \mathbf{s}_{2}+\tilde{\mathbf{w}}+\mathbf{w}
$$

where the signal-dependent noise is given by

$$
\tilde{\mathbf{w}}=\mathbf{s}_{1} \otimes \mathbf{e}_{1}+\mathbf{s}_{2} \otimes \mathbf{e}_{2}
$$

Then, the binary hypothesis test is

$$
\mathbf{z} \sim\left\{\begin{array}{l}
p_{0}(\mathbf{z})=\mathcal{C} \mathcal{N}(\mathbf{0}, \mathbf{R}) \\
p_{1}(\mathbf{z} ; \delta)=\mathcal{C N}(\delta \mathbf{p}, \mathbf{R})
\end{array}\right.
$$

where $\mathbf{R}=\sigma^{2} \mathbf{I}+\sigma_{e}^{2}\left(\mathbf{s}_{1} \mathbf{s}_{1}^{H}+\mathbf{s}_{2} \mathbf{s}_{2}^{H}\right)$. So, the same methodology can be exploited as in the above section since the formulation of the two problems is similar, i.e., a deterministic signal of interest corrupted by a white or colored Gaussian noise. The limitation of this work lies in its not enough flexible specification of the error model. In the sequel, the aim is to tackle this problem in a more generic and flexible way.

\section{B. Breaking away from existing contributions}

We think that the contributions of our work is twofold. Firstly, we consider a very generic modeling of the uncertainty on the TAD and secondly, a very popular non-Gaussian noise model is considered.

1) Random TAD: In our scenario we suppose that we do not have full knowledge of the true angular distance $\delta=\omega_{2}-\omega_{1}$ between the two sources. We only know its mean value $\delta_{0}$. To deal with this uncertainty, we model the amplitude of the vector $\mathbf{p}$ as a Gaussian random variable with mean value $\delta_{0}$ and variance $\sigma_{\delta}^{2}$, i.e.,

$$
\delta \sim \mathcal{N}\left(\delta_{0}, \sigma_{\delta}^{2}\right)
$$

We think that our modeling of the problem is more general than the two frameworks described before in the sense that a large panel of uncertainty causes can be encompassed. 
2) Compound-Gaussian noise: To generalize this scenario, in this paper we relax this hypothesis and we consider a colored compound-Gaussian (CG) noise as for instance it is standard in high resolution radar detection [19]-[21], in MIMO radar target localization [5] or in astronomical robust imaging [29,30]. The CG distribution family has many applications and is quite often used, for instance, to model the radar clutter in which targets and other interferences are embedded. With this model the vector $\mathbf{w}$ is the product between two terms, that is

$$
\mathbf{w}=\sqrt{\tau} \mathbf{x}
$$

where the positive random variable $\tau$, called texture, takes into account the local variations of the power, and the complex vector $\mathrm{x}$ called speckle is complex Gaussian distributed, i.e.,

$$
\mathbf{x} \sim \mathcal{C N}(\mathbf{0}, \mathbf{M})
$$

where $\mathbf{M}=E\left\{\mathbf{x x}^{H}\right\}$ is its covariance matrix (see for instance [19]-[21].) For ease, the speckle power is supposed equal to one, then the elements along the main diagonal of the speckle covariance matrix are $[\mathbf{M}]_{i, i}=1$. It is worth noting that $E\left\{\mathbf{w} \mathbf{w}^{H}\right\}=E\{\tau\} E\left\{\mathbf{x x}^{H}\right\}=\mu \mathbf{M}$ and $E\left\{\mathbf{w w}^{H} \mid \tau\right\}=\tau \mathbf{M}$.

3) Derivation of the expected CUB: The considered binary hypothesis test is

$$
\mathbf{z} \sim\left\{\begin{array}{l}
p_{0}(\mathbf{z} \mid \tau)=\mathcal{C N}\left(\mathbf{0}, \mathbf{M}_{0}\right), \\
p_{1}(\mathbf{z} \mid \tau)=\mathcal{C N}\left(\mathbf{0}, \mathbf{M}_{1}\right)
\end{array}\right.
$$

where $\mathbf{M}_{0}=\tau \mathbf{M}$ and $\mathbf{M}_{1}=\tau \mathbf{M}+\sigma_{\delta}^{\mathbf{2}} \mathbf{p} \mathbf{p}^{H}$. The detection theory framework cannot deal efficiently with the considered scenario since an analytical closed expression of the error probability is mathematically not tractable. So, we follow the methodology of the expected CUB introduced in a general way in the Appendix. With this model we can now derive $\Gamma(\mathbf{z} \mid \tau)$ and solve the integral in (55). It is possible to prove that

$$
\begin{aligned}
\Gamma(\mathbf{z} \mid \tau) & =\log \left(\frac{p_{1}(\mathbf{z} \mid \tau)}{p_{0}(\mathbf{z} \mid \tau)}\right)=\log \frac{\left|\mathbf{M}_{\mathbf{0}}\right|}{\left|\mathbf{M}_{\mathbf{1}}\right|}-\left(\mathbf{z}-\delta_{0} \mathbf{p}\right)^{H} \mathbf{M}_{1}^{-1}\left(\mathbf{z}-\delta_{0} \mathbf{p}\right)+\mathbf{z}^{H} \mathbf{M}_{0}^{-1} \mathbf{z} \\
& =\log \frac{\left|\mathbf{M}_{0}\right|}{\left|\mathbf{M}_{1}\right|}-\mathbf{z}^{H}\left(\mathbf{M}_{1}^{-1}-\mathbf{M}_{0}^{-1}\right) \mathbf{z}+2 \operatorname{Re}\left\{\delta_{0} \mathbf{z}^{H} \mathbf{M}_{1}^{-1} \mathbf{z}\right\}-\delta_{0}^{2} \mathbf{p}^{H} \mathbf{M}_{1}^{-1} \mathbf{p} .
\end{aligned}
$$

Using Woodbury's identity [27], we can derive that

$$
\mathbf{M}_{1}^{-1}=\mathbf{M}_{0}^{-1}-\frac{\sigma_{\delta}^{2} \mathbf{M}_{0}^{-1} \mathbf{p} \mathbf{p}^{H} \mathbf{M}_{0}^{-1}}{1+\sigma_{\delta}^{2} \mathbf{p}^{H} \mathbf{M}_{0}^{-1} \mathbf{p}}
$$

Replacing eq. (22) in (21) we obtain

$$
\Gamma(\mathbf{z} \mid \tau)=\log \frac{\left|\mathbf{M}_{0}\right|}{\left|\mathbf{M}_{1}\right|}+\frac{\sigma_{\delta}^{2}\left|\mathbf{z}^{H} \mathbf{M}_{0}^{-1} \mathbf{p}\right|^{2}}{1+\sigma_{\delta}^{2} \mathbf{p}^{H} \mathbf{M}_{0}^{-1} \mathbf{p}}+2 \operatorname{Re}\left\{\frac{\delta_{0} \mathbf{z}^{H} \mathbf{M}_{0}^{-1} \mathbf{p}}{1+\sigma_{\delta}^{2} \mathbf{p}^{H} \mathbf{M}_{0}^{-1} \mathbf{p}}\right\}-\frac{\delta_{0}^{2} \mathbf{p}^{H} \mathbf{M}_{0}^{-1} \mathbf{p}}{1+\sigma_{\delta}^{2} \mathbf{p}^{H} \mathbf{M}_{0}^{-1} \mathbf{p}}
$$

Observing that $\left|\mathbf{M}_{1}\right|=\left|\mathbf{M}_{0}\right|\left(1+\sigma_{\delta}^{2} \mathbf{p}^{H} \mathbf{M}_{0}^{-1} \mathbf{p}\right)$ and recalling that $\mathbf{M}_{0}=\tau \mathbf{M}$ we can write

$$
\Gamma(\mathbf{z} \mid \tau)=\log \frac{\tau}{\tau+a}+\frac{\sigma_{\delta}^{2}\left|\mathbf{z}^{H} \mathbf{M}_{0}^{-1} \mathbf{p}\right|^{2}}{\tau(\tau+a)}+\frac{2 \delta_{0}}{\tau+a} \operatorname{Re}\left\{\mathbf{z}^{H} \mathbf{M}_{0}^{-1} \mathbf{p}\right\}-\frac{b}{\tau+a}
$$

where $a=\sigma_{\delta}^{2} \mathbf{p}^{H} \mathbf{M}^{-1} \mathbf{p}$ and $b=\delta_{0}^{2} \mathbf{p}^{H} \mathbf{M}_{0}^{-1} \mathbf{p}$.

The key statistic that appears in the previous equation is $t=\mathbf{z}^{H} \mathbf{M}^{-1} \mathbf{p}=t_{I}+j t_{Q}$, that, conditioned on the r.v. $\tau$, is the output of a whitening matched filter [20,27], where $t_{I}=\operatorname{Re}\left\{\mathbf{z}^{H} \mathbf{M}^{-1} \mathbf{p}\right\}$ and $t_{Q}=\operatorname{Im}\left\{\mathbf{z}^{H} \mathbf{M}-1 \mathbf{p}\right\}$. 
Under the null hypothesis, and again conditioned on $\tau, E\left\{t_{I} \mid \tau, \mathcal{H}_{0}\right\}=E\left\{t_{Q} \mid \tau, \mathcal{H}_{0}\right\}=0, \operatorname{var}\left(t_{I} \mid \tau, \mathcal{H}_{0}\right)=$ $\operatorname{var}\left(t_{I} \mid \tau, \mathcal{H}_{0}\right)=\frac{\tau}{2} \mathbf{p}^{H} \mathbf{M}^{-1} \mathbf{p}$ and the two random variables $t_{I}$ and $t_{Q}$ are Gaussian distributed and independent [20,27]. With these observations we can rewrite eq. (24) as

$$
\Gamma(\mathbf{z} \mid \tau)=\log \frac{\tau}{\tau+a}+\frac{\sigma_{\delta}^{2}}{\tau(\tau+a)}\left(t_{I}+\frac{\delta_{0} \tau}{\sigma_{\delta}^{2}}\right)^{2}+\frac{\sigma_{\delta}^{2}}{\tau(\tau+a)} t_{Q}^{2}-\frac{\xi \tau}{\tau+a}-\frac{b}{\tau+a}
$$

where we define the parameter $\xi=\frac{\delta_{0}^{2}}{\sigma_{\delta}^{2}}$, that is, the ratio between the square mean value of the TAD and its variance. From (25) we obtain that

$$
E_{\mathbf{z} \mid \tau, \mathcal{H}_{0}}\{\exp (s \Gamma(\mathbf{z} \mid \tau))\}=\frac{\tau^{s}}{(\tau+a)^{s}} \exp \left(-s \frac{\xi \tau+b}{\tau+a}\right) M_{y_{1} \mid \tau, \mathcal{H}_{0}}(s) M_{y_{2} \mid \tau, \mathcal{H}_{0}}(s)
$$

where $Y_{1}\left|\tau, \mathcal{H}_{0}=\frac{\sigma_{\delta}^{2}}{\tau(\tau+a)}\left(t_{I}+\frac{\delta_{0} \tau}{\sigma_{\delta}^{2}}\right)^{2}, Y_{2}\right| \tau, \mathcal{H}_{0}=\frac{\sigma_{\delta}^{2}}{\tau(\tau+a)} t_{Q}^{2}$ and $M_{x}(s)=\exp (s x)$ is the Moment Generating Function of the random variable $X$.

We can now prove that $Y_{1} \mid \tau, \mathcal{H}_{0}$ is a scaled non-central random variable $\chi_{1}^{2}(d, \lambda)$ where $d=\frac{a}{2(\tau+a)}$ is the scale parameter and $\lambda=2 \frac{\xi \tau}{a}$ is the non-centrality parameter. Conversely $Y_{2} \mid \tau, \mathcal{H}_{0}$ is a scaled central $\chi_{1}^{2}(d)$ random variable with the same scale parameter. Now, we are able to derive $M_{y_{1} \mid \tau, \mathcal{H}_{0}}(s)$ and $M_{y_{2} \mid \tau, \mathcal{H}_{0}}(s)$ according to

$$
\begin{gathered}
M_{y_{1} \mid \mathcal{H}_{0}}(s)=\frac{1}{\sqrt{1-\frac{a}{\tau+a} s}} \exp \left(\xi \frac{\tau}{\tau+a} \frac{s}{\left(1-\frac{a}{\tau+a} s\right)}\right) \\
M_{y_{2} \mid \mathcal{H}_{0}}(s)=\frac{1}{\sqrt{1-\frac{a}{\tau+a} s}}
\end{gathered}
$$

Replacing (27) and (28) in (26) we obtain

$$
E_{\mathbf{z} \mid \tau, \mathcal{H}_{0}}\{\exp (s \Gamma(\mathbf{z} \mid \tau))\}=\frac{\tau^{s}}{(\tau+a)^{s}} \exp \left(\xi \frac{\tau}{\tau+a} \frac{s}{\left(1-\frac{a}{\tau+a} s\right)}\right) \exp \left(-s \frac{\xi \tau+b}{\tau+a}\right) \frac{1}{1-\frac{a}{\tau+a} s} .
$$

Replacing now (29) in (53) we finally get the CUB

$$
P_{e} \leq \frac{1-\alpha}{\beta^{s}} E_{\tau}\left\{\frac{\tau^{s}}{(\tau+a)^{s}} \exp \left(\xi \frac{\tau}{\tau+a} \frac{s}{\left(1-\frac{a}{\tau+a} s\right)}\right) \exp \left(-s \frac{\xi \tau+b}{\tau+a}\right) \frac{1}{1-\frac{a}{\tau+a} s}\right\}
$$

The final value of the CUB for the CG noise depends on the PDF that we choose for modeling the texture $\tau$.

In order to vary the uncertainty on the value of the TAD, we have defined the parameter $\xi=\frac{\delta_{0}^{2}}{\sigma_{\delta}^{2}}$. For low values of $\xi$ the variance of the TAD is large, so the uncertainty is high. Conversely, for high values of $\xi$ the uncertainty is small.

Define now the random variable $\rho=\frac{\tau}{a+b}$. Observing that $\xi=b / a$, it results that $\tau=\frac{b(1+\xi)}{\xi} \rho$, then for the mean value $E_{\tau}\{\cdot\}$ in eq. 30 we can write

$$
E_{\tau}\{\cdot\}=E_{\rho}\left\{\frac{[(1+\xi) \rho]^{s}}{[(1+\xi) \rho+1]^{s-1}} \frac{1}{(1+\xi) \rho+1-s} \exp \left[\frac{\xi s(s-1)}{(1+\xi) \rho+(1-s)}\right]\right\}
$$


It is apparent from the previous equation that $E_{\rho}\{\cdot\}$ does not depend on $\delta_{0}^{2}$ and $\sigma_{\delta}^{2}$ separately, but only on their ratio $\xi$.

If $\sigma_{\delta}^{2}=0$, that is the TAD is deterministic, $a=0, \xi \rightarrow \infty$, and the CUB reduces to

$$
P_{e} \leq \frac{1-\alpha}{\beta^{s}} E_{\rho}\left\{\exp \left[\frac{s}{\rho}(-1+s)\right]\right\}
$$

a) Gaussian noise: The Gaussian distribution is a particular case of the compound-Gaussian family. As a matter of fact, if the noise is Gaussian distributed with power $\sigma^{2}$, the probability density function of the variable $\tau$ is a delta of Dirac centered in $\tau=\sigma^{2}$, then, from eq. (30) the probability of error satisfies the inequality

$$
P_{e} \leq \frac{1-\alpha}{\beta^{s}} \frac{\left(\sigma^{2}\right)^{s}}{\left(\sigma^{2}+a\right)^{s}} \frac{1}{1-s \frac{a}{\sigma^{2}+a}} \cdot \exp \left[-\frac{s}{\sigma^{2}+a}\left(\xi \sigma^{2}+b\right)\right] \exp \left[-\frac{\xi \sigma^{2} s}{\sigma^{2}+a} \frac{1}{\left(1-s \frac{a}{\sigma^{2}+a}\right)}\right]
$$

For ease, let's define SNR at the output of the whitening matched filter $t=\mathbf{z}^{H} \mathbf{M}^{-1} \mathbf{p}$.

$$
S N R=\frac{E\left\{\left|\delta \mathbf{p}^{H} \mathbf{M}^{-1} \mathbf{p}\right|^{2}\right\}}{E\left\{\left|\mathbf{d}^{H} \mathbf{M}^{-1} \mathbf{p}\right|^{2}\right\}}=\frac{\left(\delta_{0}^{2}+\sigma_{\delta}^{2}\right)}{\sigma^{2}} \mathbf{p}^{H} \mathbf{M}^{-1} \mathbf{p}=\frac{a+b}{\sigma^{2}}=\gamma
$$

Observing that, with the definition (34) $\sigma^{2}=b(\xi+1) /(\xi \gamma)$ we can rewrite eq. (33) as

$$
P_{e} \leq \frac{1-\alpha}{\beta^{s}} \frac{(1+\xi)^{s}}{[1+\xi+\gamma]^{s}} \frac{1}{1-s \frac{\gamma}{1+\xi+\gamma}} \exp \left[-\frac{\xi \gamma(1-s) s}{1+\xi+\gamma(1-s)}\right]
$$

It is now evident that the CUB does not depend on $\delta_{0}^{2}$ and $\sigma_{\delta}^{2}$ separately, but only on their ratio $\xi$.

For low values of SNR, $\gamma<<1$. Under this hypothesis eq. (33) can be approximated as

$$
P_{e} \leq \frac{1-\alpha}{\beta^{s}} \exp \left[\frac{s \xi \gamma}{1+\xi}(-1+s)\right]
$$

and the value of $s$ for which the bound is minimum is $s=\frac{1}{2}+\frac{1+\xi}{2 \xi \gamma} \log (\beta)$. When $\operatorname{Pr}\left(\mathcal{H}_{0}\right)=\operatorname{Pr}\left(\mathcal{H}_{1}\right), \beta=1$ and $s_{\min }=\frac{1}{2}$.

Conversely, when $\gamma>>1$, eq. (33) can be approximated as

$$
P_{e} \leq \frac{1-\alpha}{\beta^{s}} \frac{(1+\xi)^{s}}{\gamma^{s}} \frac{1}{(1-s)} \exp [-s \gamma]
$$

and $s_{\text {min }}=1-\frac{1}{\xi+\log (\beta \gamma /(1+\xi))}$.

In the general case $s_{\text {min }}$, the value of $s$ that minimizes the CUB, can be obtained first calculating the $\log C U B$ and then derivating it with respect to $s$. After some calculations it turns out that $s_{\min }$ can be calculated as the unique solution in the range $(0,1)$ of the 2 nd order equations

$$
s^{2}-\left[\frac{\gamma^{2}-2 K_{0} \gamma(1+\xi+\gamma)}{K_{0} \gamma^{2}}\right] s+\frac{K_{0}(1+\xi+\gamma)^{2}-(1+\xi+\gamma)[(1+\xi) \xi+\gamma]}{K_{0} \gamma^{2}}=0
$$

where $K_{0}=\left[\xi-\log \frac{(1+\xi)}{\beta(1+\xi+\gamma)}\right]$.

In the purely deterministic case of $\delta=\delta_{0}, a=0, \gamma=b / \mu$ and $\xi \rightarrow \infty$, then 


$$
P_{e} \leq \frac{1-\alpha}{\beta^{s}} \exp [\gamma s(-1+s)]
$$

and the value of $s$ for which the bound is minimum is $s=\frac{1}{2}+\frac{1}{2 \gamma} \log (\beta)$. When $\beta=1$ again $s_{\text {min }}=\frac{1}{2}$.

b) K-distributed noise: For analyzing the behavior of the Chernoff Bound in a CG general case, we used the $\mathrm{K}$ model, a particular case of the Compound-Gaussian family, characterized by a Gamma-distributed texture $\tau$, then

$$
p(\tau)=\frac{1}{\Gamma(\nu)}\left(\frac{\nu}{\mu}\right)^{\nu} \tau^{\nu-1} \exp \left(-\frac{\nu}{\mu} \tau\right) u(\tau)
$$

The parameter $\nu$ characterizes the spikyness of the noise: the lower $\nu$, the spikier the K-distributed noise [20,21]. For $\nu \rightarrow \infty$ the $\mathrm{K}$ distribution degenerates into the Rayleigh one. The parameter $\mu$ represents the power of the noise. Recalling eq.(31) and that $\rho=\frac{\xi}{b(1+\xi)} \tau$, with a Gamma-distributed texture, the PDF of $\rho$ is given by

$$
p(\rho)=\frac{1}{\Gamma(\nu)}\left(\frac{\nu}{\mu}\right)^{\nu}\left[\frac{b(1+\xi)}{\xi}\right]^{\nu} \rho^{\nu-1} \exp \left(-\frac{\nu}{\mu} \frac{b(1+\xi)}{\xi} \rho\right) u(\rho)
$$

Since the power of the noise is defined by the parameter $E\{\tau\}=\mu$, the $S N R$ is now $\frac{a+b}{\mu}=\gamma$. Then, similarly to the Gaussian case, $\mu=b(\xi+1) /(\xi \gamma)$. Replacing this expression in eq. (41) yields to

$$
p(\rho)=\frac{1}{\Gamma(\nu)}(\nu \gamma)^{\nu} \rho^{\nu-1} \exp (-\nu \gamma \rho) u(\rho)
$$

The new expression of $p(\rho)$ depends only on the shape parameter $\nu$ and on the signal-to-noise power ratio $\gamma$. As a consequence eqs. (31) and (30) depends only on $\nu, \gamma$ and $\xi$. As for the Gaussian case, the CUB does not depend on $\delta_{0}^{2}$ and $\sigma_{\delta}^{2}$ separately, but only on their ratio $\xi$.

In the purely deterministic case of $\delta=\delta_{0}, a=0, \gamma=b / \mu$ and $\xi \rightarrow \infty$, then from 32

$$
C U B=\frac{1-\alpha}{\beta^{s}} E_{\rho}\left\{\exp \left[\frac{s}{\rho}(-1+s)\right]\right\}=\frac{1-\alpha}{\beta^{s}} \frac{2}{\Gamma(\nu)}(\nu \gamma s(1-s))^{\nu / 2} K_{\nu}(2 \sqrt{\gamma s(1-s) \nu})
$$

To find the $s$ for which the "deterministic" CUB is the tightest we can calculate the $\log C U B$ and then derivate with respect to $s$, that is

$\frac{\partial \log C U B}{\partial s}=-\log \beta+\frac{\nu}{2} \frac{(1-2 s)}{s(1-s)}+\frac{1}{K_{\nu}(z)} \frac{\partial K_{\nu}(z)}{\partial z} \frac{\partial z}{\partial s}=-\log \beta+\frac{\nu}{2} \frac{(1-2 s)}{s(1-s)}+\frac{(1-2 s)}{K_{\nu}(z)} \frac{\partial K_{\nu}(z)}{\partial z} \sqrt{\frac{\gamma \nu}{s(1-s)}}$

where $z=2 \sqrt{\gamma s(1-s) \nu}$. If $\beta=1$, the only solution of eq. 44 is $s=1 / 2$. Then, for equiprobable hypotheses and deterministic ARL, the tightest bound is the Batthacharya Upper Bound (BUB) [17],

$$
B U B=\frac{1}{2 \Gamma(\nu)}(\gamma \nu)^{\frac{\nu}{2}} K_{\nu}(\sqrt{\gamma})
$$

whatever $\nu, b$ and $\mu$. 


\section{AnAlysis OF RESUlts}

In the following figures 1-5 we show the $s_{\min }$ as a function of the SNR for Gaussian $(\mathrm{G})$ and K-distributed noise (K) with varying values of $\nu, \beta=1$ (the two hypotheses are equipropable) and different values of the parameter $\xi$. As expected for low values of SNR, in the Gaussian case $s_{\min }=0.5$, while, in the $\mathrm{K}$ noise case, $s_{\min }>0.5$, with increasing values corresponding to increased spikyness (i.e. decreasing $\nu$ ).

It is worth observing that the behavior of $s_{\min }$ highly depends on $\xi$. To highlight the dependence of the CUB on the parameter $\xi$, in figure 6 we show the $P_{e}$ as a function of $\xi$ for $S N R=30 \mathrm{~dB}$. The curves for all tested values of $\nu$ are quite close. It is worth noting that in our example for $\xi=0.5 \sigma_{\delta}^{2}=0.1$, while for $\xi=10 \sigma_{\delta}^{2}=10^{-3}$ and the TAD can be almost considered deterministic. This figure shows that, ignoring the randomness of $\delta$, we can make a largely wrong prediction on the system performance, even of some order of magnitude in term of $P_{e}$.

To complete our analysis in figure 7 we set $\sigma_{\delta}^{2}=0.1$ and we show the SNR necessary for guaranteeing $P_{e}=10^{-3}$ as a function of $\delta_{0}$. Looking at this figure we can conclude that, even for a small variance, while for resolving two sources whose average distance is $\delta_{0}=1$ only $20 \mathrm{~dB}$ are necessary, for an average angular distance of $\delta_{0}=0.1$, more than $40 \mathrm{~dB}$ are required, for both Gaussian and K-distributed clutter. For a certain fixed $\sigma_{\delta}^{2}, \delta_{0}$ in this figure can be interpreted in the same way as the statistical angular resolution limit ARL of the case of deterministic unknown TAD. It provides the average TAD that guarantees the fixed detection performance. As expected, it depends on the $P_{e}$ and the SNR.

\section{CONClusions}

Usually, the limit in terms of angular resolution for array processing is studied based on the hypothesis that the angular separation is unknown and deterministic. In the context of a convenient associated binary hypothesis test, the distribution of the GLRT for an asymptotic number of measurements/sensors can be characterized in closedform. This allows to derived the TAD with respect to the SNR, the array manifold and a non-centrality parameter parameterized by the probabilities of false alarm and detection. In realistic context, the knowledge on the array manifold or on the sources is often uncertain. Based on this observation, we adopt a generic and flexible error model on the TAD by considering it as a Gaussian random variable, i.e., $\delta \sim \mathcal{N}\left(\delta_{0}, \sigma_{\delta}\right)$. The degree of randomness (or uncertainty) is deduced and quantified by the ratio $\xi=\delta_{0}^{2} / \sigma_{\delta}^{2}$. The usual asymptotic characterization of the GLRT is no longer adapted to take into account efficiently a general error cause. To cope with this limitation, we adopt an alternative strategy based on the Chernoff Upper Bound (CUB) on the error probability. In this work, the CUB is extended to take into account nuisance parameters since we assume that the background noise follows a compound Gaussian model. This model is very popular in many signal-based applications as for instance radar robust processing or robust imaging for astronomical data. Our derivations allow to propose analytic closed-forms of the CUB and the BUB (Batthacharya Upper Bound). In particular, we show that the SNR to resolve two closely-spaced sources for a given error probability is highly dependent on the average TAD. 


\section{APPENDIX: CUB With RANDOM NUISANCE PARAMETERS}

\section{A. The Bayes' detection theory}

Let $\operatorname{Pr}\left(\mathcal{H}_{i}\right)$ be the a priori hypothesis probability with $\operatorname{Pr}\left(\mathcal{H}_{0}\right)+\operatorname{Pr}\left(\mathcal{H}_{1}\right)=1$. Let $\operatorname{Pr}\left(\mathbf{y} \mid \mathcal{H}_{i}\right)$ and $\operatorname{Pr}\left(\mathcal{H}_{i} \mid \mathbf{y}\right)$ be the $i$-th conditional hypothesis and the posterior probabilities, respectively. The Bayes' detection rule chooses the hypothesis $\mathcal{H}_{i}$ associated with the largest posterior probability $\operatorname{Pr}\left(\mathcal{H}_{i} \mid \mathbf{y}\right)$. Introduce the indicator hypothesis function according to $\phi(\mathbf{y}) \sim \operatorname{Bernou}(\alpha)$ where $\operatorname{Bernou}(\alpha)$ stands for the Bernoulli distribution of success probability $\alpha=\operatorname{Pr}(\phi(\mathbf{y})=1)=\operatorname{Pr}\left(\mathcal{H}_{1}\right)$. Function $\phi(\mathbf{y})$ is defined on $\mathcal{X} \rightarrow\{0,1\}$ where $\mathcal{X}$ is the data-set of cardinality $|\mathcal{X}|$ enjoying the following decomposition $\mathcal{X}=\mathcal{X}_{0} \cup \mathcal{X}_{1}$ where $\mathcal{X}_{0}=\{\mathbf{y}: \phi(\mathbf{y})=0\}=\mathcal{X} \backslash \mathcal{X}_{1}$ and

$$
\mathcal{X}_{1}=\{\mathbf{y}: \phi(\mathbf{y})=1\}=\left\{\mathbf{y}: \Omega(\mathbf{y})=\log \frac{\operatorname{Pr}\left(\mathcal{H}_{1} \mid \mathbf{y}\right)}{\operatorname{Pr}\left(\mathcal{H}_{0} \mid \mathbf{y}\right)}>0\right\}
$$

where $\Omega(\mathbf{y})$ is the $\log$ posterior-odds ratio. Let $\varepsilon$ be a random nuisance parameter unconditioned with respect to the hypothesis in set $\Omega$, i.e., $p_{i}(\varepsilon)=p\left(\varepsilon \mid \mathcal{H}_{i}\right)=p(\varepsilon)$. The average probability of error is

$$
P_{e}=E_{\mathbf{y}}\{\operatorname{Pr}(\text { Error } \mid \mathbf{y})\}=E_{\mathbf{y}} E_{\boldsymbol{\varepsilon} \mid \mathbf{y}}\{\operatorname{Pr}(\text { Error } \mid \mathbf{y}, \boldsymbol{\varepsilon})\}
$$

with

$$
\operatorname{Pr}(\text { Error } \mid \mathbf{y}, \boldsymbol{\varepsilon})=\left\{\begin{array}{lll}
\operatorname{Pr}\left(\mathcal{H}_{0} \mid \mathbf{y}, \boldsymbol{\varepsilon}\right) & \text { if } & \mathbf{y} \in \mathcal{X}_{1}, \\
\operatorname{Pr}\left(\mathcal{H}_{1} \mid \mathbf{y}, \boldsymbol{\varepsilon}\right) & \text { if } & \mathbf{y} \in \mathcal{X}_{0} .
\end{array}\right.
$$

The standard strategy to minimize $\operatorname{Pr}($ Error $\mid \mathbf{y}, \boldsymbol{\varepsilon})$ for a given $\mathbf{y}$ is $\min \left\{\operatorname{Pr}\left(\mathcal{H}_{0} \mid \mathbf{y}, \boldsymbol{\varepsilon}\right), \operatorname{Pr}\left(\mathcal{H}_{1} \mid \mathbf{y}, \boldsymbol{\varepsilon}\right)\right\}$ [27]. So using (47), the minimal average error probability can be expressed according to

$$
P_{e}=E_{\mathbf{y}, \varepsilon}\left\{\min \left\{\operatorname{Pr}\left(\mathcal{H}_{0} \mid \mathbf{y}, \varepsilon\right), \operatorname{Pr}\left(\mathcal{H}_{1} \mid \mathbf{y}, \varepsilon\right)\right\}\right\}
$$

Using the Bayes' relation and the statistical independence of $\varepsilon$, we have

$$
\operatorname{Pr}\left(\mathcal{H}_{i} \mid \mathbf{y}, \boldsymbol{\varepsilon}\right)=g_{i}(\mathbf{y} ; \varepsilon) f(\mathbf{y}, \boldsymbol{\varepsilon})
$$

where $g_{i}(\mathbf{y} ; \boldsymbol{\varepsilon})=p_{i}(\mathbf{y} \mid \varepsilon) \operatorname{Pr}\left(\mathcal{H}_{i}\right)$ is conditioned to the hypothesis and $f(\mathbf{y}, \boldsymbol{\varepsilon})=\frac{p(\boldsymbol{\varepsilon})}{p(\mathbf{y}, \boldsymbol{\varepsilon})}$ is unconditioned. This implies that $f(\mathbf{y}, \varepsilon)$ plays no role in the minimization of eq. (49). Thus,

$$
\begin{aligned}
P_{e} & =\int_{\Omega} \int_{\mathcal{X}} \min \left\{g_{0}(\mathbf{y} ; \boldsymbol{\varepsilon}), g_{1}(\mathbf{y} ; \boldsymbol{\varepsilon})\right\} f(\mathbf{y}, \boldsymbol{\varepsilon}) p(\mathbf{y}, \boldsymbol{\varepsilon}) \mathrm{d} \mathbf{y} \mathrm{d} \boldsymbol{\varepsilon} \\
& =E_{\boldsymbol{\varepsilon}} \int_{\mathcal{X}} \min \left\{(1-\alpha) p_{0}(\mathbf{y} \mid \boldsymbol{\varepsilon}), \alpha p_{1}(\mathbf{y} \mid \boldsymbol{\varepsilon})\right\} \mathrm{d} \mathbf{y}
\end{aligned}
$$

\section{B. Chernoff Upper Bound (CUB)}

Using the property [17] that $\min \{a, b\} \leq a^{s} b^{1-s}$ with $a, b>0$ and $s \in(0,1)$ in (52) the minimal error probability is upper bounded according to

$$
P_{e} \leq \frac{1-\alpha}{\beta^{s}} E_{\varepsilon}\left\{\exp \left[-C_{\mathbf{y} \mid \boldsymbol{\varepsilon}}(s)\right\}\right]
$$

where $\beta=\frac{1-\alpha}{\alpha}$ and

$$
C_{\mathbf{y} \mid \boldsymbol{\varepsilon}}(s)=-\log \int_{\mathcal{X}} p_{0}(\mathbf{y} \mid \varepsilon)^{1-s} p_{1}(\mathbf{y} \mid \varepsilon)^{s} \mathrm{~d} \mathbf{y}
$$


is the (Chernoff) $s$-divergence. Term $C_{\mathbf{y} \mid \varepsilon}(s)$ characterizes the exponential rate of the error exponent of $P_{e}$. The Chernoff information, denoted by $C_{\mathbf{y} \mid \boldsymbol{\varepsilon}}(s)$, is a characterization on the best achievable Bayes' error probability [17]. It is worth observing that the integral in (54) can be reformulated as

$$
\begin{aligned}
\int_{\mathcal{X}} p_{0}(\mathbf{y} \mid \varepsilon)^{1-s} p_{1}(\mathbf{y} \mid \varepsilon)^{s} \mathrm{~d} \mathbf{y} & =\int_{\mathcal{X}} \frac{p_{1}(\mathbf{y} \mid \varepsilon)^{s}}{p_{0}(\mathbf{y} \mid \varepsilon)^{s}} p_{0}(\mathbf{y} \mid \varepsilon) \mathrm{d} \mathbf{y}=\int_{\mathcal{X}} \exp [s \Gamma(\mathbf{y} \mid \varepsilon)] p_{0}(\mathbf{y} \mid \varepsilon) \mathrm{d} \mathbf{y} \\
& =E_{\mathbf{y} \mid \boldsymbol{\varepsilon}, \mathcal{H}_{0}}\{\exp (s \Gamma(\mathbf{y} \mid \varepsilon))\}=M_{\Gamma\left(\mathbf{y} \mid \varepsilon, \mathcal{H}_{0}\right)}(s)
\end{aligned}
$$

where $\Gamma(\mathbf{y} \mid \varepsilon)=\log \left(\frac{p_{1}(\mathbf{y} \mid \varepsilon)}{p_{0}(\mathbf{y} \mid \varepsilon)}\right)$, and $M_{X}(s)$ is the Moment Generating Function (MGF) of the random variable $X$.

\section{REFERENCES}

[1] S. T. Smith, "Statistical resolution limits and the complexified Cramér-Rao bound," IEEE Trans. on Signal Processing, 53 :1597-1609, May 2005.

[2] A. Amar and A.J.Weiss, "Fundamental limitations on the resolution of deterministic signals," IEEE Trans. on Signal Processing, 56(11) :5309-5318, November 2008 .

[3] A. Amar and A.J. Weiss, "Fundamental resolution limits of closely spaced random signals, " IET Radar, Sonar \& Navigation, Volume 2, No. 3, 2008, pp. 170-179.

[4] Z. Liu and A. Nehorai, "Statistical angular resolution limit for point sources," IEEE Trans. on Signal Processing, 55(11) :5521-5527, November 2007.

[5] X. Zhang, M. N. El Korso, and M. Pesavento. "MIMO radar target localization and performance evaluation under SIRP clutter." Signal Processing, 130 (2017): 217-232.

[6] R. Boyer, "Performance Bounds and Angular Resolution Limit for the Moving Co-Located MIMO Radar," IEEE Transactions on Signal Processing, Volume 59, No. 4, 2011, pp. 1539-1552.

[7] M. Thameri, K. Abed-Meraim, F. Foroozan, R. Boyer, and A. Asif, "On the Statistical Resolution Limit (SRL) for Time-Reversal based MIMO radar," Signal Processing, Volume 144, March 2018, pp. 373-383

[8] M. N. El Korso, R. Boyer,, A. Renaux and S. Marcos, "Statistical Resolution Limit for Source Localization With Clutter Interference in a MIMO radar Context," IEEE Transactions on Signal Processing, Volume 60, No. 2, Feb. 2012, pp. 987-992.

[9] M.N. El Korso, R. Boyer, A. Renaux, and S. Marcos, "Statistical Resolution Limit of the Uniform Linear Cocentered Orthogonal Loop and Dipole Array" , IEEE Transactions on Signal Processing, Volume 59, No. 1, 2011, pp. 425-431.

[10] M. N. El Korso, R. Boyer, A. Renaux and S. Marcos, "On the Asymptotic Resolvability Of Two Point Sources in Known Subspace Interference Using a GLRT-Based Framework" , Signal Processing, Volume: 92, Issue: 10, Oct. 2012, pp. 2471-248

[11] M. N. El Korso, R. Boyer, A. Renaux and S. Marcos, "Statistical Resolution Limit for the Multidimensional Harmonic Retrieval Model : Hypothesis Test and Cramér-Rao Bound Approaches" , EURASIP Journal on Advances in Signal Processing, special issue "Advances in Angle-of-Arrival and Multidimensional Signal Processing for Localization and Communications" , No. 12, 2011, pp. 1-14.

[12] N.D. Tran, R. Boyer, S. Marcos and P. Larzabal, "On The Angular Resolution Limit For Array Processing In The Presence Of Modeling Errors," IEEE Transactions on Signal Processing, Volume : 61, No. 19, Oct. 2013, pp. 4701-4706.

[13] M. Shahram and P. Milanfar, "On the resolvability of sinusoids with nearby frequencies in the presence of noise," IEEE Trans. on Signal Processing, vol. 53, no. 7, pp. 2579-2585, Jul. 2005.

[14] S. M. Kay, Fundamentals of Statistical Signal Processing : Detection Theory, NJ: Prentice Hall, 1998, vol. 2.

[15] M.A. Richards, Fundamentals of Radar Signal Processing, McGraw Hill, 2005.

[16] F. Nielsen, "An information-geometric characterization of Chernoff information," IEEE Signal Processing Letters, vol. 20, no. 3, pp. 269-272, 2013.

[17] T. M. Cover and J. A. Thomas, Elements of Information Theory. New York, NY, USA : Wiley-Interscience, 1991.

[18] S. Sinanovic and D. H. Johnson, "Toward a theory of information processing," Signal Processing, vol. 87, no. 6, pp. $1326-1344,2007$. 
[19] F. Gini, M. Greco, M. Diani, and L. Verrazzani, "Performance Analysis of Two Adaptive Radar Detectors against Non-Gaussian Real Sea Clutter Data," IEEE Transactions on Aerospace and Electronic Systems, Vol. 36, No. 4, October 2000, pp. 1429-1439.

[20] F. Gini, M. Greco and A. Farina, "Clairvoyant and Adaptive Signal Detection in Non-Gaussian Clutter: a Data-Dependent Threshold Interpretation,” IEEE Trans. on Signal Processing, Vol. 47, No. 6, pp. 1522-1531, June 1999.

[21] F. Gini and M. Greco, "A Suboptimum Approach to Adaptive Coherent radar Detection in Compound-Gaussian Clutter," IEEE Transactions on Aerospace and Electronic Systems, Vol. 35, No. 3, pp. 1095-1104, July 1999.

[22] M. Greco, F. Gini. A. Farina, "Joint Use of Sum and Delta Channels for Multiple Radar Target DOA Estimation,'IEEE Transactions on Aerospace and Electronic Systems, Vol. 43, No. 3, July 2007.

[23] C.D. Austin, , J. N. Ash, and R.L. Moses, "Dynamic dictionary algorithms for model order and parameter estimation" IEEE Transactions on Signal Processing, Vol. 61, No. 20, pp. 5117-5130, 2013

[24] G.-T. Pham, R. Boyer and F. Nielsen. "Computational Information Geometry for Binary Classification of High-Dimensional Random Tensors". MDPI Entropy, MDPI, 2018, 20 (3).

[25] R. Pribic. "Information Distances in Stochastic Resolution Analysis" International Conference on Geometric Science of Information. Springer, Cham, 2017.

[26] R. Pribic and G. Leus. "Information distances for radar resolution analysis." IEEE Computational Advances in Multi-Sensor Adaptive Processing (CAMSAP), 2017.

[27] S. M. Kay, Fundamentals of statistical signal processing : estimation theory, PTR Prentice-Hall, Englewood Cliffs, NJ, 1993.

[28] X. Zhang, M. N. El Korso, and M. Pesavento, "MIMO Radar Performance Analysis under K-distributed Clutter", in Proc. of IEEE International Conference on Acoustics, Speech, and Signal Processing (ICASSP), 2014.

[29] V. Ollier, M. N. El Korso, A. Ferrari, R. Boyer and P. Larzabal, "Robust distributed calibration of radio interferometers with direction dependent distortions," Signal Processing, Vol. 153, 2018, Pp 348-354.

[30] V. Ollier, M. N. El Korso, A. Ferrari, R. Boyer and P. Larzabal. "Bayesian calibration using different prior distributions: an iterative maximum a posteriori approach for radio interferometers" EUSIPCO, Sep. 2018, Rome, Italy.

[31] P. Stoica, J. Li, and Y. Xie, "On probing signal design for MIMO radar." IEEE Transactions on Signal Processing, vol. 55, no. 8, pp. 4151-4161, 2007.

[32] S.J. Wijnholds, S. van der Tol, R. Nijboer and A.-J. van der Veen, "Calibration challenges for future radio telescopes", IEEE Signal Process. Mag., vol. 27, pp. 30-42, 2010. 


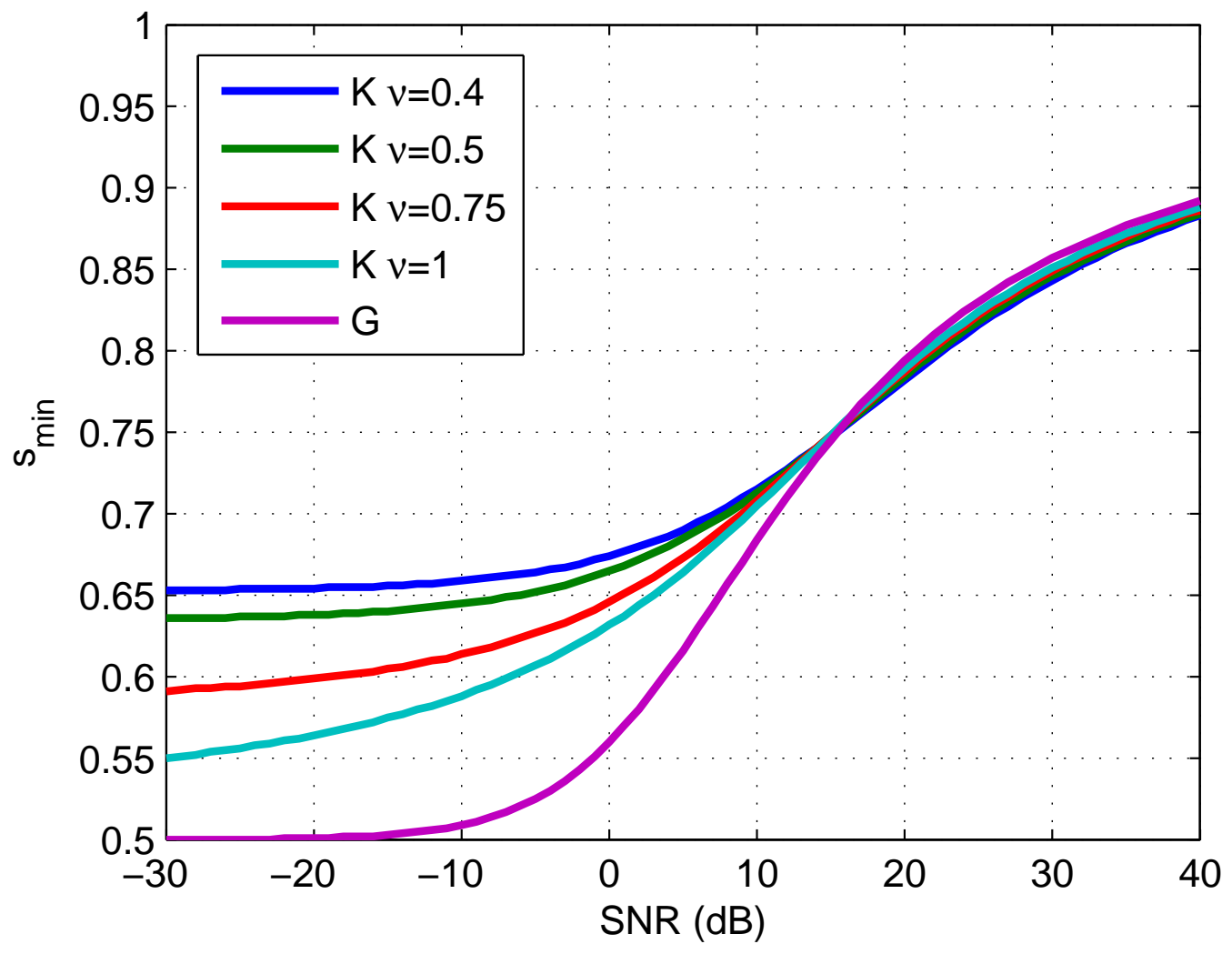

Fig. 1. $s_{\min }$ vs SNR for K and Gaussian noise, $\xi=0.25, \beta=1$ 


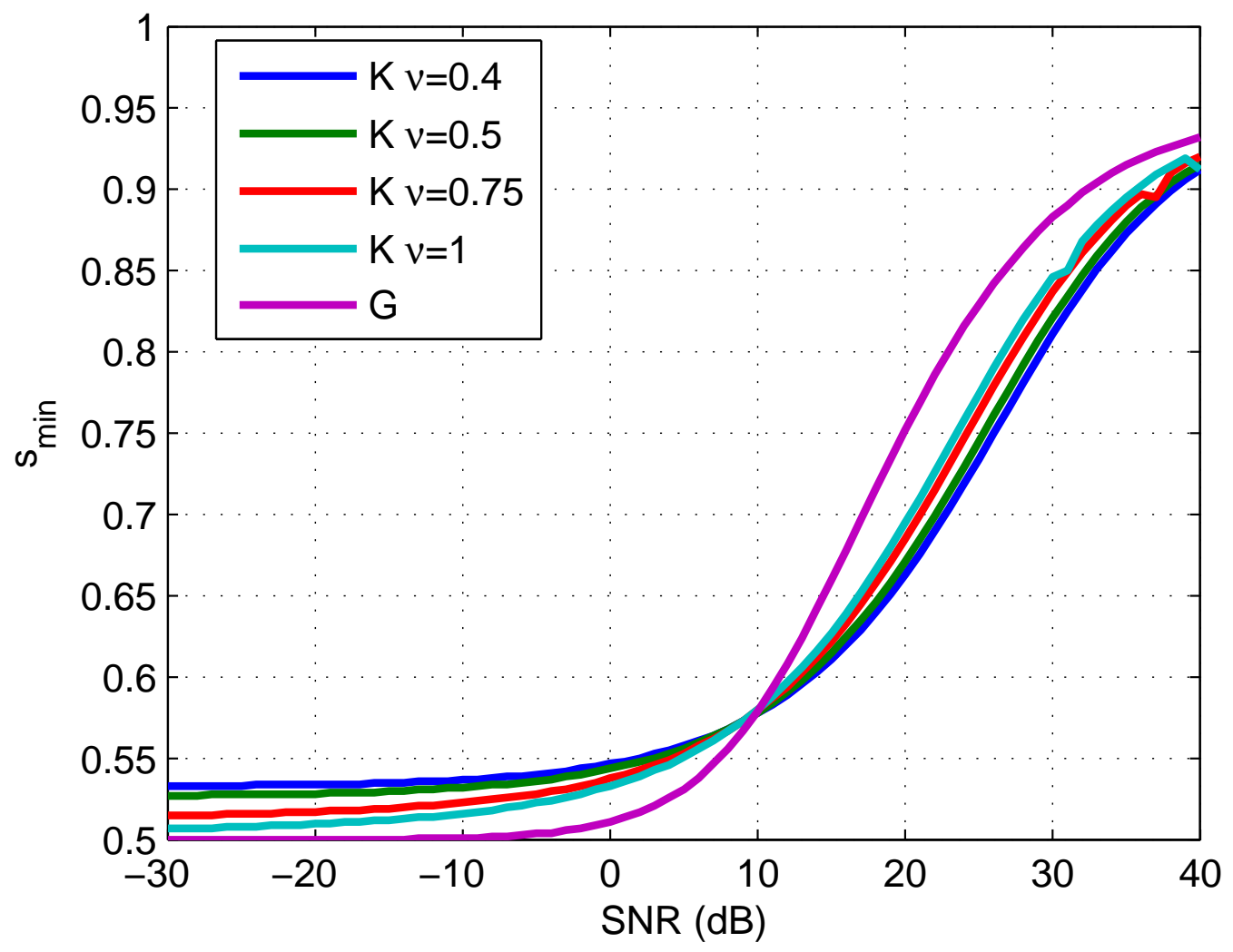

Fig. 2. $s_{\min }$ vs SNR for $\mathrm{K}$ and Gaussian noise, $\xi=10, \beta=1$ 


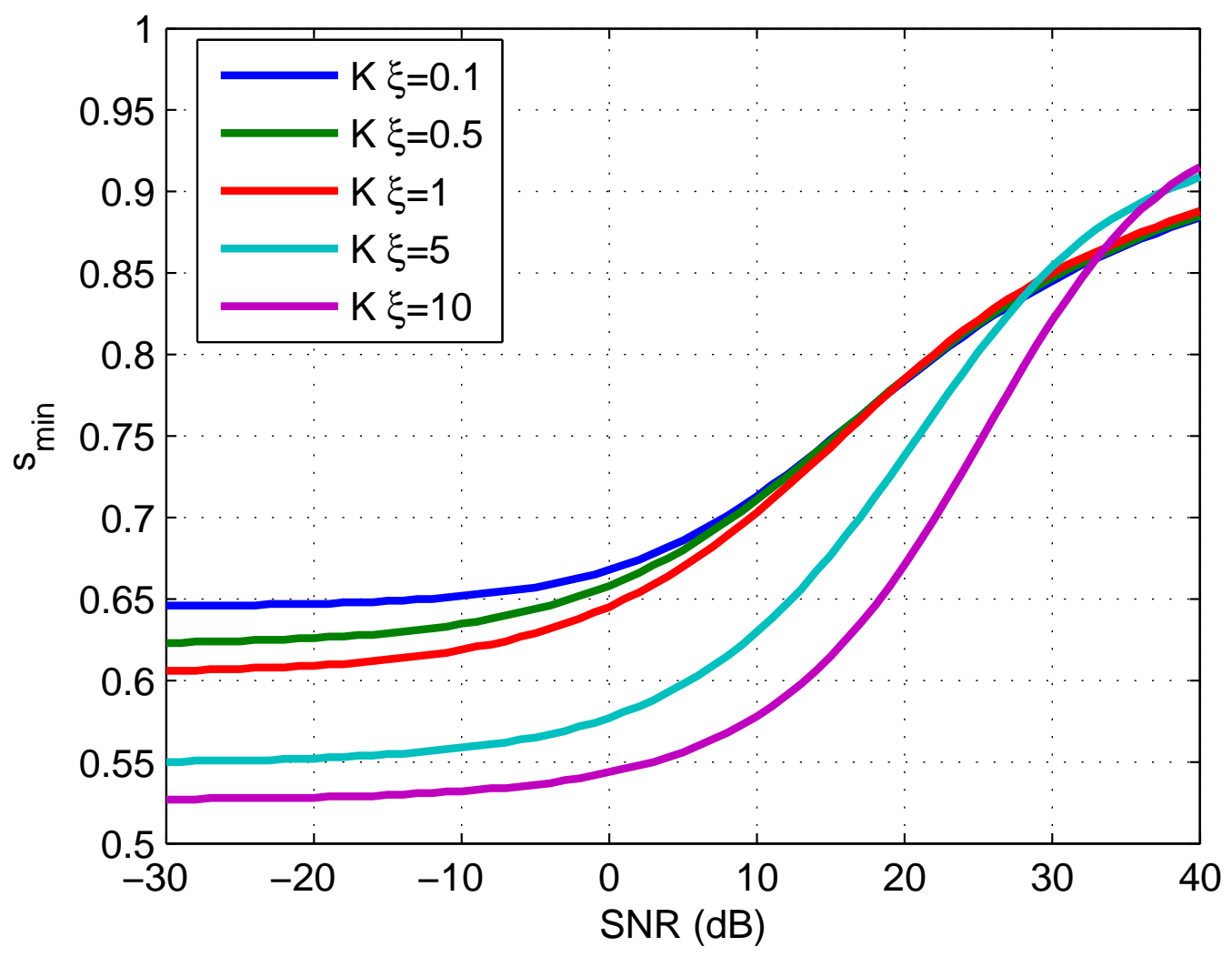

Fig. 3. $s_{\min }$ vs SNR for K noise, $\nu=0.5, \beta=1$ 


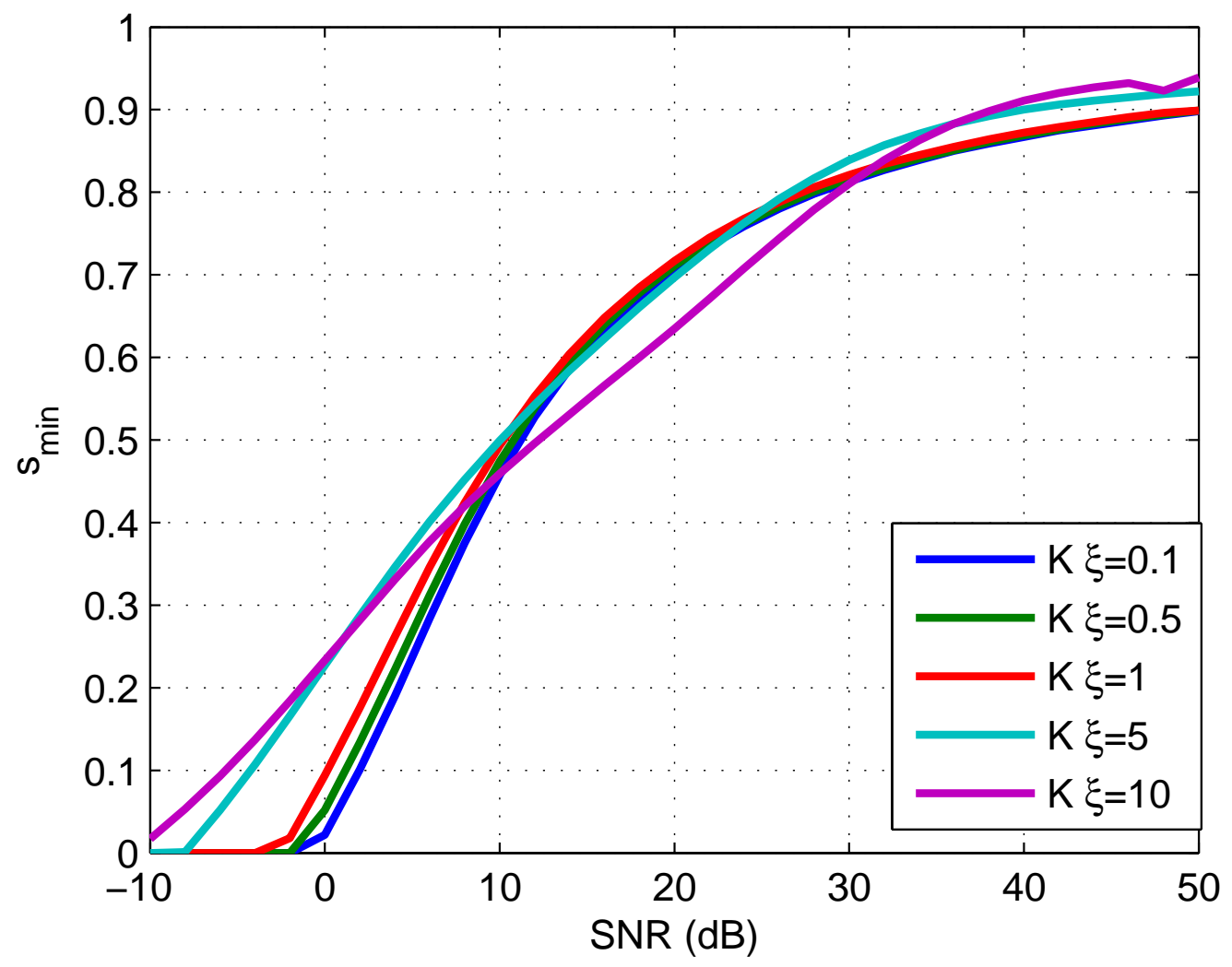

Fig. 4. $s_{\min }$ vs SNR for $\mathrm{K} \nu=0.5, \beta=0.333$ 


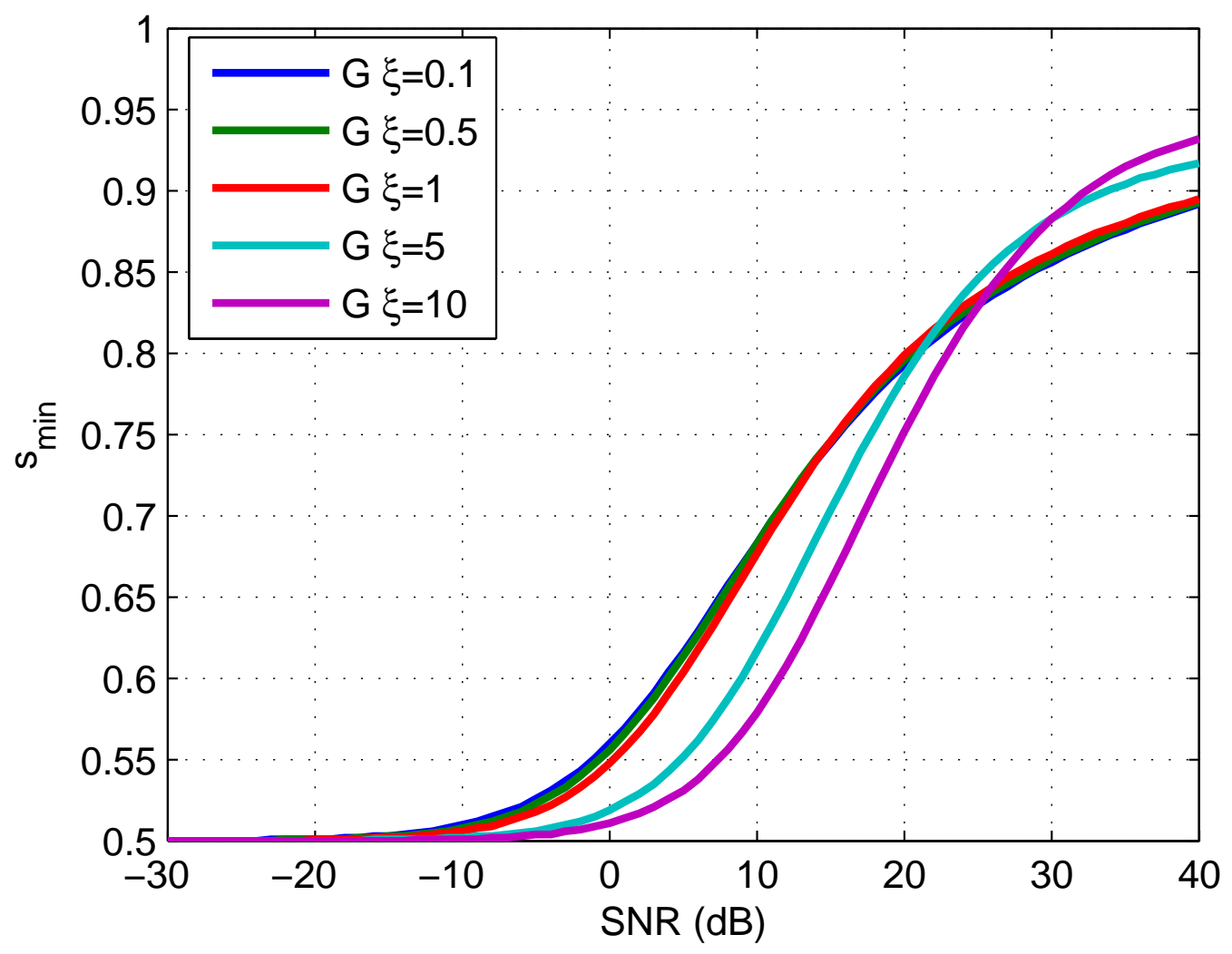

Fig. 5. $s_{\text {min }}$ vs SNR for Gaussian noise, $\beta=1$ 


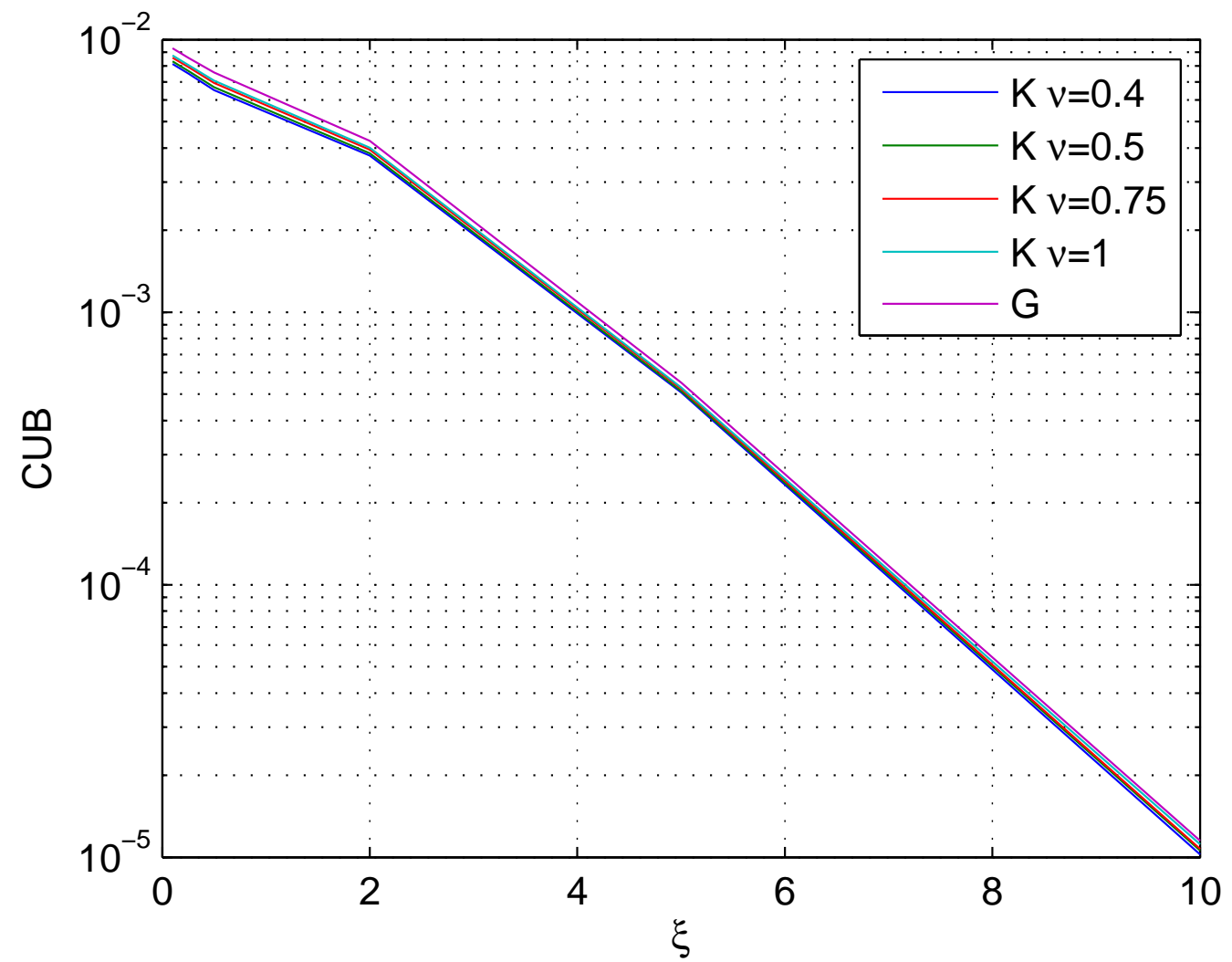

Fig. 6. CUB vs $\xi$ for $\mathrm{K}$ and Gaussian noise, $S N R=30 d B, \beta=1$ 


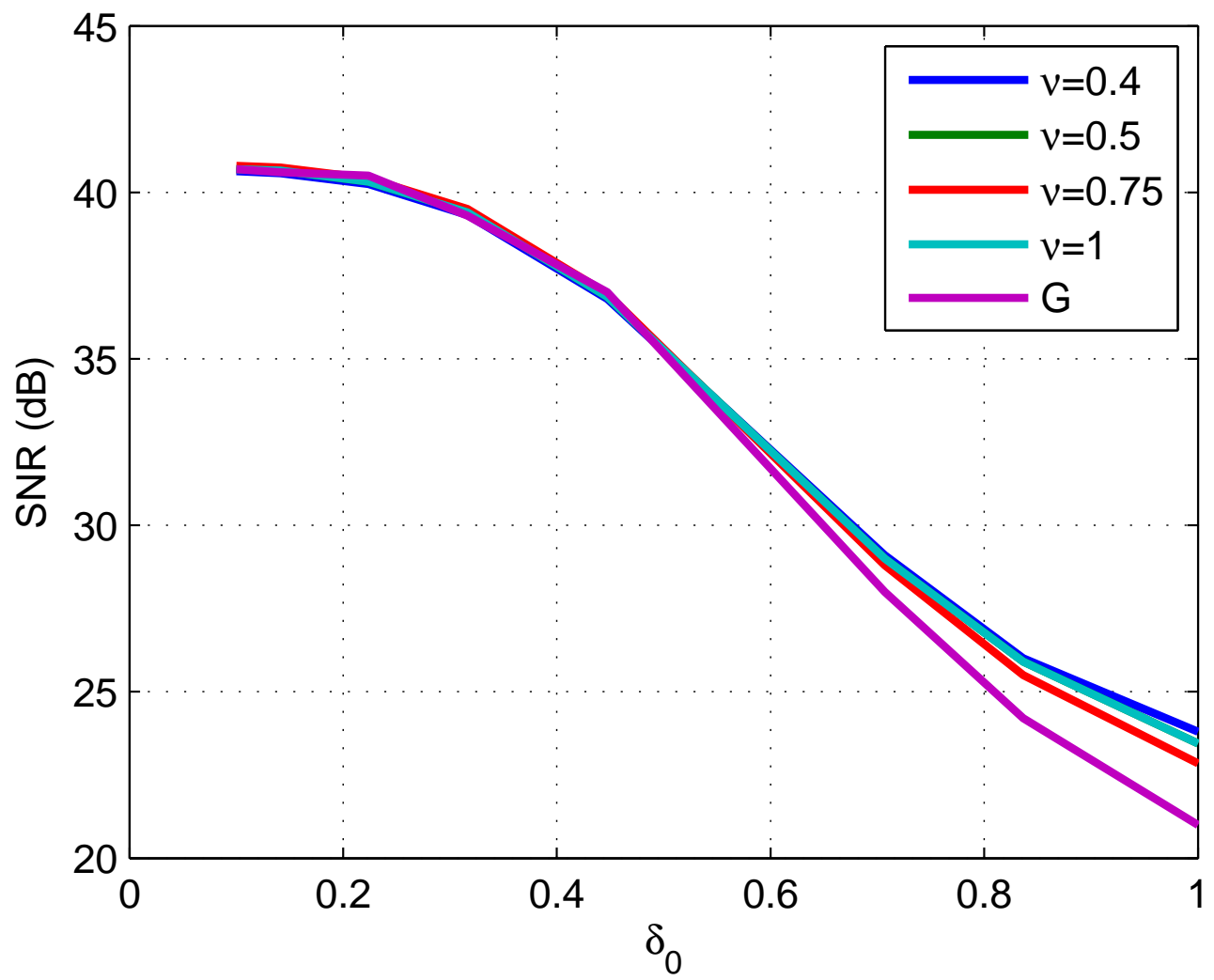

Fig. 7. SNR vs $\delta_{0}$ for $\mathrm{K}$ and Gaussian noise, $\sigma_{\delta}^{2}=0.1, \beta=1$ 